\title{
The Geometry Asymmetry of Mean Pulse Profile of Pulsar
}

\author{
X.J. $\mathrm{Wu}^{1,2}$, J. $\mathrm{Pan}^{1,2}$ and X.B. $\mathrm{Xu}^{1,2}$ \\ ${ }^{1}$ Beijing Astrophysics Center, CAS-PKU, Beijing 100871, China \\ ${ }^{2}$ Astronomy Department, Peking University, Beijing 100871, China
}

\begin{abstract}
In this paper, the effects of aberration, retardation and magnetic field sweep back, which are caused by pulsar's rapid rotation, are estimated to see their influences on the pulse shape of pulsars and geometry asymmetry of the mean pulse profile. The width of mean pulse profile is modified only slightly, but the geometry asymmetry appears and will exhibit different behaviors at different cases. The pulsars of B1451-68 and J0437-4715 are analyzed as examples.
\end{abstract}

\section{Introduction}

The rotating vector model (RVM) of RC69 has been the standard model used to interpret pulsars mean profile and the polarization assuming that the emission are emitted along the dipole magnetic field lines. Theoretical modifications of the RVM including aberration, retardation and MFS (P92 and S83, S85). The total longitude shift $(\delta \phi)$ of the emission at altitude $\mathrm{r}$ relative to $\mathrm{r}=0$ is

$$
\delta \phi=\delta \phi_{a}+\delta \phi_{r}-\delta \phi_{m f s} .
$$

The phase shift $\delta \phi>0$ expresses that the emission of the component will received earlier than what RVM predicts, while $\delta \phi<0$ indicates delay. The $\delta \phi_{a}$ is aberration of the center of the beam. At altitude $\mathrm{r}$ the shift of phase by aberration is $\delta \phi_{a}=\tan ^{-1}\left(\gamma r / r_{L C}\right)$. In which $\beta=\Omega r(\sin \alpha) / c$ and $\gamma=\left(1-\beta^{2}\right)^{-1 / 2}$. If $r<<r_{L C}, \gamma \simeq 1$. The $\delta \phi_{r}$ is retardation, which is purely a propagation effect. Suppose emission of a frequency is emitted at altitude $r$, then there will be a longitude shift relative to $r=0, \delta \phi_{r}=r / r_{L C}$. The MFS is caused by pulsar rotational energy loss in the form of magnetic dipole radiation. The electromagnetic torque on the neutron star results in a toroidal bending of the magnetic field opposite to the sense of the star's rotation (S83, S85). At the altitude $r$ the phase shift caused by MFS is

$$
\delta \phi_{m f s} \simeq 1.2 \sin ^{2} \alpha\left(r / r_{L C}\right)^{3}
$$

There are a group of relations between total longitude shift $(\delta \phi)$ and emission height at different magnetic inclination angle $(\alpha)$. A turning point exist on curve with samll $\alpha\left(\leq 50^{\circ}\right)$ at altitude of $r_{p}$. The $r_{p}$ is an important new parameter.

\section{Geometry Asymmetry of Mean Pulse Profile of Pulsar}

After the work of R93a and R93b, it is generally recognized that there exist three components in beam: core, inner cone and outer cone, which locate at different height. They exhibit a five component mean pulse profile. The $P_{1}, P_{2}$, $P_{3}, P_{4}$ and $P_{5}$ are the pulse phase of the component's peak, and $w_{1}, w_{2}, w_{3}$, 
$w_{4}$, and $w_{5}$ are the half width of components. Three parameters describing the asymmetry of mean profile can be defined as below:

$$
\begin{gathered}
\lambda_{1}=\left(P_{3}-\left(P_{1}-w_{1} / 2\right)\right) /\left(\left(P_{5}+w_{5} / 2\right)-P_{3}\right) \\
\lambda_{2}=\left(P_{3}-\left(P_{2}-w_{2} / 2\right)\right) /\left(\left(P_{4}+w_{4} / 2\right)-P_{3}\right) \\
\lambda_{3}=\left(\left(P_{2}-w_{2} / 2\right)-\left(P_{1}-w_{1} / 2\right)\right) /\left(\left(P_{5}+w_{5} / 2\right)-\left(P_{4}+w_{4} / 2\right)\right)
\end{gathered}
$$

Under the assumption of symmetry of the structure of emission components without aberration, retardation and MFS, the core component would locate exactly at the middle point of the mean profile and $\lambda_{1}=\lambda_{2}=\lambda_{3}=1$. Suppose that outer cone, inner cone and core are emitted at altitudes of $r_{0}, r_{i}$, and $r_{c}$, the phase shifts (Eq.(1)) are $\delta \phi_{0}, \delta \phi_{i}, \delta \phi_{c}$, the asymmetry parameters can be calculated.

\section{Geometry Asymmetry of Mean Pulse Profile}

When $r<r_{p}$ for the core and two cones, the phase shift increases with the increasing of $\mathrm{r}$. The PSRB1451-68 is in this case, $\lambda_{1}=1.04, \lambda_{2}=1.07$ and $\lambda_{3}=1.02$ at $400 \mathrm{MHz} ; \lambda_{1}=1.31, \lambda_{2}=1.02$ and $\lambda_{3}=1.35$ at $950 \mathrm{MHz}$. The profile parameters used from Wu98. That is normal configuration, their asymmetry parameters are all greater than 1. It means that $r_{0}>r_{i}>r_{c}$ for them and all of $r_{0}, r_{i}, r_{c}$ is less than $r_{p}$.

When $r>r_{p}$, the phase shift decreases with the increasing of $r$. For the ms pulsar PSRJ0437-4715 with period 0.00567s, its radius of light cylinden is small, than its conal emission height $r / r_{L C}$ may be comparable with its $r_{p} / r_{L C} \approx 0.5$. It is possible that the height of outer cone is larger than the $r_{p}$, and $\lambda_{3}<1$. The PSRJ0437-4715 is in this case. The calculated results are $\lambda_{1}=0.99, \lambda_{2}=1.32$, $\lambda_{3}=0.65$ at $90 \mathrm{MHz} ; \lambda_{1}=1.31, \lambda_{2}=1.80$ and $\lambda_{3}=0.87$ at $430 \mathrm{MHz}$. The profile parameters used from M95.

Acknowledgments. This work is supported by NSF of China, the Climbing Program from the state Science and Technology Commission of China, and by RFDP Of China.

\section{References}

Manchester, R.N. \& Simon, J. 1995, ApJ, 441, L65 (M95)

Philips, J.A. 1992, ApJ, 385, 282 (P92)

Radhakrishnan, V. \& Cooke, D.J. 1969, ApJ, 3, 225 (RC69)

Rankin, J.M. 1993, ApJSS, 85, 145 (R93b)

Rankin, J.M. 1993, ApJ, 405, 285 (R93a)

Shitov, Yu.P. 1983, Soviet Astron., 27, 314 (S83)

Shitov, Yu.P. 1985, Soviet Astron., 29, 33 (S85)

Wu, X.J, Gao, X.Y., Rankin, J.M., Xu, W. \& Malofeev, V.M. 1998, AJ, 116, 1984 (Wu98) 